\section{PS6:110 MOTOR AND COGNITIVE FATIGUE IN SLE IS ASSOCIATED WITH MOOD AND HEALTH-RELATED QUALITY OF LIFE (HRQOL) IN PATIENTS WITH SLE: RESULTS FROM THE PATIENT REPORTED OUTCOMES IN LUPUS (PRO-LUPUS) STUDY}

${ }^{1} \mathrm{~A}$ Bland, ${ }^{2} \mathrm{~S}$ Hunziker ${ }^{3,4} \mathrm{M}$ Barraclough, ${ }^{5} \mathrm{~K}$ Kane, ${ }^{6} \mathrm{R}$ Cervera, ${ }^{7} \mathrm{~T} D$ Döner, ${ }^{8} \mathrm{~A} D$ D ${ }^{9} \mathrm{~J}$ Pouchot, ${ }^{10} \mathrm{~T}$ Stoll, ${ }^{11,12} \mathrm{I}-\mathrm{K}$ Penner, ${ }^{3,4} \mathrm{IN}$ Bruce. ${ }^{1}$ Division of neuroscience and experimental psychology, The University of Manchester, Manchester, UK; ${ }^{2}$ University of Basel, Basel, Switzerland; ${ }^{3}$ Aruk Centre for Epidemiology, CFMDR, FMBH, The University of Manchester, MAHSC, Manchester, UK; ${ }^{4}$ The Kellgren Centre for Rheumatology, NIHR Manchester BRC, Manchester University Hospitals NHS FT, MAHSC, Manchester, UK; ${ }^{5}$ Division of Musculoskeletal and Dermatological Sciences, The University of Manchester, Manchester, UK; ${ }^{6}$ Department of Autoimmune Diseases, Hospital Clinic, Barcelona, Spain; ${ }^{7}$ Department Medicine/Rheumatology and Clinical Immunology, Charite Universitätsmedizin Berlin, GERMANY; ${ }^{8}$ Rheumatology Unit, Department of Medicine, University of Padova, Italy; ${ }^{9}$ Internal Medicine Department, European Georges Pompidou Hospital, Paris Descrates University, Paris, FRANCE; ${ }^{10}$ Klinik fur Rheumatologie Geriatrie und Rehabilitation, Spituler Schaffhausen, Schaffhausen, SWITZERLAND; "Zentrum für Angewandte Neurokognition und Neuropsychologische Forschung, COGNITO, Düsseldorf, Germany; ${ }^{12}$ Klinik für Neurologie, Heinrich Heine Universität, Düsseldorf, Germany

\subsection{6/lupus-2018-abstract.154}

Background Fatigue is a commonly reported problem in systemic lupus erythematosus (SLE) but the cause and impact of this symptom are not fully understood. Clinical, physical and psychosocial aspects may affect fatigue as well as cognitive performance. Of note, the influence of disease status and inflammatory disease activity on fatigue in SLE is poorly understood. We aimed to better understand these associations by studying changes in fatigue over time.

Methods 100 SLE patients, all with lupus nephritis, were recruited across 5 European countries. Participants completed assessments examining; fatigue (FSMC: Fatigue Scale for Motor and Cognitive functions), disease activity (SLEDAI, tender joints score), SLICC damage index, cognition (SPRT: Spatial Recall Test, SDMT: Symbol Digits Modalities Test, PASAT: Paced Auditory Serial Addition Test, SRT: Selective Reminding Test, WLG: Word List Generation), pain (pain mannequin), depression (CES-D: Centre for Epidemiological Studies-Depression Scale), steroid dose and HRQoL (SF-36 v2, LupusQoL), at baseline and at follow-up, 3-4 months later. Correlations of score changes between baseline and follow-up were calculated using SPSS 22 and any significant results $(\mathrm{p}<0.01)$ are reported below.

Results All participants met 1997 revised ACR criteria for SLE and had biopsy-proven lupus nephritis (ISN grades II-V). The mean (SD) SLEDAI score was 4.67 (5.05) at baseline and 4.37 (5.55) at follow-up. Cognitive scores were significantly different at baseline compared to follow-up, where all scores improved. When examining the correlations between changes in score from baseline to follow-up, there were significant associations between changes in fatigue (motor and cognitive) scores with mood, the physical subscale of the SF-36 and a number of domains of the LupusQOL including pain and burden to others (Table 1). No significant correlations were found between changes in fatigue and disease activity, damage, steroid dose or cognitive measures.

Conclusions In SLE patients the major associations of motor and cognitive fatigue are with mood, pain and poorer HRQoL. We found no significant correlations with disease activity, damage or steroid dose. Self-rated QoL and depression may more directly impact fatigue than disease activity and these psychosocial aspects need to be addressed to help manage fatigue more effectively in SLE.

\section{PS6:111 FORTY YEARS OF A LONDON LUPUS CLINIC - A RETROSPECTIVE ANALYSIS OF DRUG USE}

BS Bernstein, T Mcdonnell, C Wincup, B Tejera Segura, A Rahman. Centre for Rheumatology, UCL, London, UK

\subsection{6/lupus-2018-abstract.155}

Background The SLE Clinic at University College London Hospital (UCLH) has been running since 1978. During this period there have been major changes in the management of SLE, including successive introduction of cyclophosphamide, mycophenolate and rituximab, and increasing recognition of the adverse effects of corticosteroids and the benefits of hydroxychloroquine. We therefore carried out a retrospective analysis of use of drugs to treat patients with SLE in this clinic, particularly looking for patterns of usage that might vary with age at diagnosis, ethnicity, gender and decade of diagnosis.

Methods Medical records from 171 patients (Mean age at diagnosis $=30,93 \%$ female, mean follow-up 22 years) were assessed to see which drugs had ever been prescribed, according to the following outcome categories - any corticosteroids, higher dose corticosteroids (intravenous or $>5 \mathrm{mg}$ per day oral), hydroxychloroquine, immunosuppressants (IS) (including mycophenolate, azathioprine, cyclophosphamide, rituximab).

Abstract PS6:110 Table 1 Significant correlative $(p<0.01)$ associated with change in score between baseline and follow-up

\begin{tabular}{lcc}
\hline Study measure & FSMC-Cognitive & FSMC-Motor \\
\hline SF-36 v2 Physical subscale & -0.365 & -0.381 \\
LupusQoL Physical subscale & -0.416 & -0.359 \\
LupusQoL Pain subscale & -0.568 & -0.514 \\
LupusQoL Burden subscale & -0.317 & -0.337 \\
LupusQoL Emotional subscale & -0.317 & $n s$ \\
LupusQoL Fatigue subscale & -0.423 & -0.316 \\
LupusQoL Planning subscale & $n s$ & -0.324 \\
LupusQoL Health subscale & $n s$ & $n s$ \\
CES-D & 0.347 & 0.335 \\
\hline
\end{tabular}

FSMC-Cognitive: Fatigue scale for motor and cognitive functions, cognitive subscale; FSMC-Motor: Fatigue scale for motor and cognitive functions, motor subscale; CES-D: Center for epidemiological studies - depression scale; SF-36: 36-item short form health survey; LupusQoL: Lupus quality of life questionnaire 
We also looked at use of higher dose corticosteroids or IS in the first two years after diagnosis. We assessed whether each of these patterns of drug use varied between different patient groups.

Results Figure 1a shows that $134(78 \%)$ of patients received steroids, 121 (71\%) hydroxychloroquine and 98 (57\%) IS. About a third (59 or 35\%) received all three. Conversely, 33 (19\%) received no drugs or hydroxychloroquine alone. 94\% of those who received IS also got steroids.

There were no significant associations with gender or ethnicity except that a higher proportion of non-white patients than white received both higher dose corticosteroids and IS $(36 \%$ vs $21 \% \mathrm{p}=0.05)$. Figure $1 \mathrm{~b}$ shows that use of most drugs was similar across all ages of onset, except that those diagnosed at $>50$ years had lower use of higher dose corticosteroids (40\%) and azathioprine (10\%) but higher use of cyclophosphamide $(30 \%)$ than other groups.

Figure 1c shows a shift to increased early use of IS and higher dose corticosteroids in patients diagnosed in more recent decades. Cyclophosphamide use has continued despite introduction of rituximab and mycophenolate.

Conclusion This long-term retrospective analysis shows that over the course of disease $81 \%$ of patients received corticosteroids and/or immunosuppressants. Early aggressive immunosuppression has become more common.

\section{PS6:112 ACCEPTANCE AND COMMITMENT THERAPY IN LUPUS, A CASE CONTROL STUDY}

${ }^{1} \mathrm{M}$ Sahebari, ${ }^{2} \mathrm{~A}$ Ahmadi, ${ }^{2} \mathrm{HR}$ Aghamohammadian, ${ }^{2} \mathrm{MJ}$ Asghari, ${ }^{1} \mathrm{SZ}$ Mirfeizi. ${ }^{1}$ Rheumatic Diseases Research Centre, Mashhad University of Medical Sciences, School of Medicine, Mashhad, Iran; ${ }^{2}$ Ferdowsi University of Mashhad, Faculty of Education and Psychology, Mashhad, Iran

10.1136/lupus-2018-abstract.156

The aim of this study was to evaluate the influence of acceptance and commitment therapy (ACT) in lupus, on frustration,
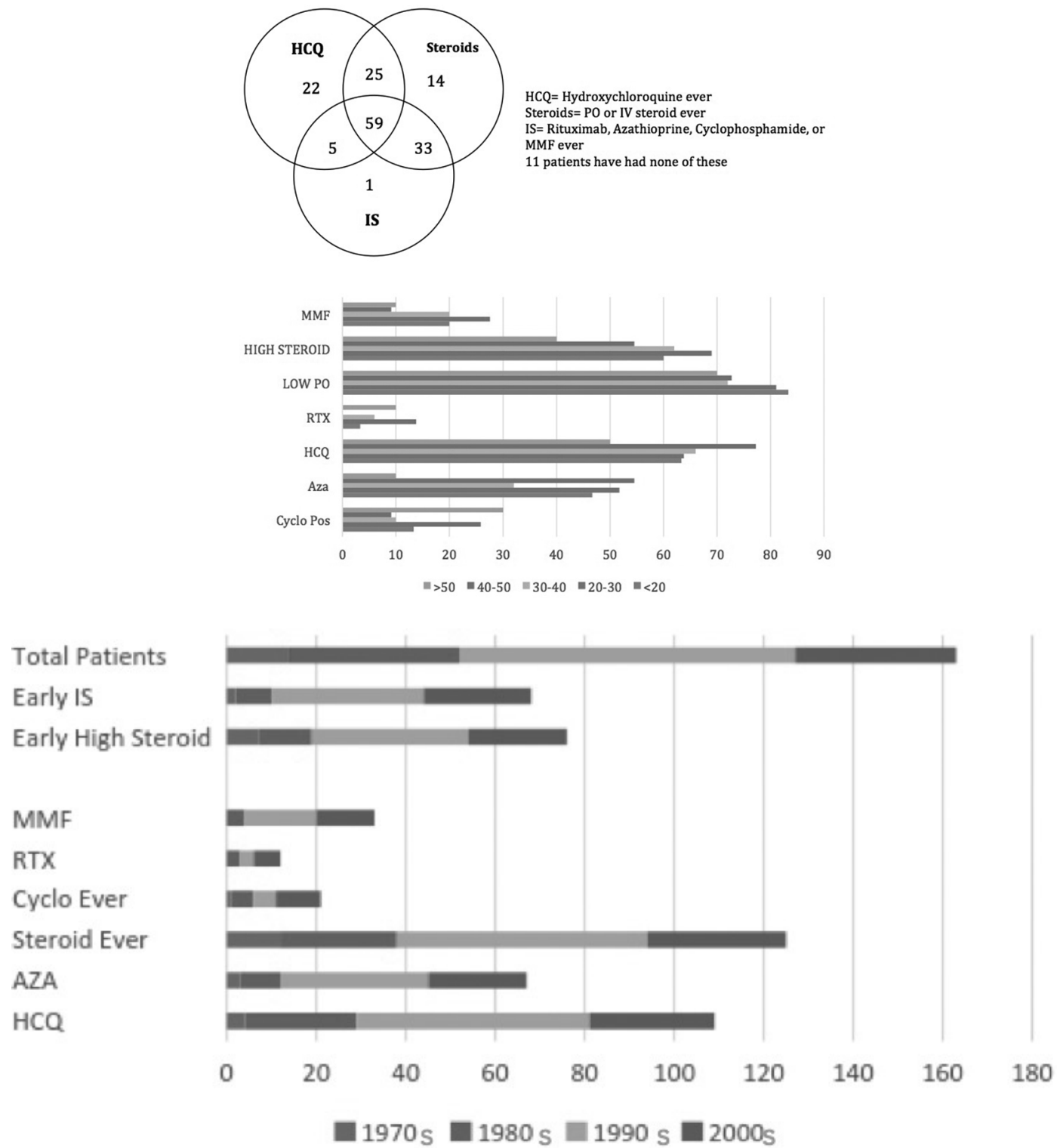

Abstract PS6:111 Figure 1 A: all drugs. B: drugs stratified by age of SLE diagnosis. C: drugs stratified by decade of diagnosis 